\title{
Fictitious forces and electronic energy relaxation in nanocrystals
}

\author{
Ho-Soon Yang, Michael R. Geller* \\ Department of Physics and Astronomy, University of Georgia, Athens, GA 30602-2451, USA
}

\begin{abstract}
It is well known that a localized electronic impurity state couples to the vibrational modes of an insulating nanoparticle through the ordinary electron-phonon interaction, enhanced by the effects of quantum confinement. In this paper we show that an electron can also interact with the translational degrees of freedom of a nanoparticle. The form of the coupling is dictated by Einstein's equivalence principle and is independent of the ordinary electron-phonon interaction. We calculate the rate of electronic energy relaxation provided by this mechanism and establish its experimental observability. (C) 1999 Elsevier Science B.V. All rights reserved.
\end{abstract}

Keywords: Nanoparticles; Nanocrystals; Energy relaxation; Fictitious forces

The dominant mechanism for the low-energy population decay of a localized electronic impurity state in a macroscopic crystal at low temperature is usually one-phonon emission [1]. In a nanocrystal, however, the reduced dimensionality causes a suppression of the phonon density of states at low energies. In particular, in a spherical nanoparticle of diameter $d$ there will be an acoustic phonon frequency gap $\Delta \omega$ of the order of $2 \pi v / d$, where $v$ is a characteristic sound velocity of the bulk crystal. The one-phonon energy relaxation rate of an electron in the excited state of a two-level system with energy spacing $\Delta \varepsilon$ therefore vanishes when $\Delta \varepsilon$ is less than $\hbar \Delta \omega$. Indeed, a considerable suppression of this relaxation rate has been observed recently in $\mathrm{Y}_{2} \mathrm{O}_{3}: \mathrm{Eu}^{3+}$ nanocrystals [2].

\footnotetext{
* Corresponding author. Fax: 706-542-2492.

E-mail address: mgeller@hal.physast.uga.edu (M.R. Geller)
}

In this paper we propose a new nonradiative relaxation mechanism caused by the inertial coupling of the electron to the nanoparticle's translational center-of-mass (CM) motion. This interaction is present because an electron bound to an impurity center in an oscillating nanoparticle is in an accelerating reference frame, and, in accordance with Einstein's equivalence principle, it feels a fictitious time-dependent force. The effect of this fictitious force is observable because the energy relaxation it provides is resonant in nature, and, as we shall show, can be quite large for a nanoparticle with weakly damped translational motion.

In an isolated nanoparticle with stress-free boundary conditions the three $\mathrm{CM}$ translational modes would have zero frequency, as there would be no restoring forces. However, any mechanical coupling to a substrate or to other nanoparticles provides a restoring force and gives the CM modes a finite frequency. For simplicity, we shall assume that the nanoparticle is subjected to a 
one-dimensional harmonic oscillator potential $V=\frac{1}{2} M \Omega^{2} X^{2}$ with frequency $\Omega$. This will lead to an effective electric field acting on the localized electron. ${ }^{1}$ Rotational motion, which would lead to an effective magnetic field, will be ignored.

We turn now to a brief summary and discussion of our results; a complete analysis shall be given in a future publication. The Hamiltonian for the nanoparticle is

$$
\begin{aligned}
H= & \sum_{\alpha} \varepsilon_{\alpha} c_{\alpha}^{\dagger} c_{\alpha}+\sum_{n} \hbar \omega_{n} a_{n}^{\dagger} a_{n}+\hbar \Omega b^{\dagger} b \\
& +\sum_{n \alpha \alpha^{\prime}} g_{n \alpha \alpha^{\prime}} c_{\alpha}^{\dagger} c_{\alpha^{\prime}}\left(a_{n}+a_{n}^{\dagger}\right)-g \sum_{\alpha \alpha^{\prime}} x_{\alpha \alpha^{\prime}} c_{\alpha}^{\dagger} c_{\alpha^{\prime}}\left(b+b^{\dagger}\right) .
\end{aligned}
$$

The first term in Eq. (1) is the Hamiltonian for the noninteracting two-level system. Here $\varepsilon_{\alpha}$ (with $\alpha=1,2$ ) are the energy levels of the localized impurity state; $c_{\alpha}^{\dagger}$ and $c_{\alpha}$ are electron creation and annihilation operators. (The electrons are taken to be spinless for simplicity.) The second term in Eq. (1) describes the nanoparticle's internal vibrational dynamics. The $\omega_{n}$ are the frequencies of the internal modes and the $a_{n}^{\dagger}$ and $a_{n}$ are the corresponding phonon creation and annihilation operators. For the case of a perfectly spherical nanoparticle the vibrational eigenmodes and eigenvalues can be obtained analytically [3]; the frequency of the lowest internal mode (a 5-fold degenerate torsional mode) is approximately $2 \pi v_{t} / d$, where $v_{t}$ is the transverse sound velocity and $d$ is the diameter.

The third term in Eq. (1) describes the harmonic dynamics of the CM. As discussed above, the nanoparticle is assumed to be constrained to move in the $x$ direction only. Hence, the CM translational motion is described by a single bosonic degree of freedom,

$b \equiv \sqrt{\frac{M \Omega}{2 \hbar}}\left(X+\frac{\mathrm{i}}{M \Omega} P\right)$,

where $M$ is the nanoparticle mass, and $X$ and $P$ are the $x$-components of the CM position and conju-

\footnotetext{
${ }^{1}$ Strictly speaking, the fictitious force couples to the mass of the electron, not its charge, and cannot be regarded as an electric field.
}

gate momentum. The fourth term in Eq. (1) is the ordinary leading-order interaction between the two-level system and the internal vibrational modes. Here $g_{n \alpha \alpha^{\prime}}$ is the electron-phonon coupling; it depends on the detailed microscopic structure of the nanoparticle, the nature and position of the impurity, and the spatial dependence of the internal vibrational modes.

The last term in Eq. (1), which describes the coupling between the two-level system and the CM motion, is the focus of the present work. Here $x_{\alpha \alpha^{\prime}} \equiv\left\langle\phi_{\alpha}|x| \phi_{\alpha^{\prime}}\right\rangle$ are dipole-moment matrix elements, which, of course, depend on the form of the impurity states $\phi_{\alpha}(\boldsymbol{r})$, and

$g \equiv \sqrt{\frac{m^{2} \Omega^{3} \hbar}{2 M}}$

is a coupling constant that depends only on the electron mass $m$ and on macroscopic properties of the nanoparticle. The last term in Eq. (1) is present because the Hamiltonian for the noninteracting two-level system, the first term in Eq. (1), is written in a coordinate system moving with the oscillating nanoparticle, which is a noninertial reference frame. An electron therefore sees an additional uniform force

$\boldsymbol{F}_{\text {eff }}=-m \ddot{\boldsymbol{R}}$,

where $\boldsymbol{R}$ is the nanoparticle CM. For the case of harmonic motion, Eq. (4) can be written as $\boldsymbol{F}_{\text {eff }}=m \Omega^{2} \boldsymbol{R}$. Thus, the potential energy of an electron at position $\boldsymbol{r}$ (in the CM frame) is

$U \equiv-m \Omega^{2} \boldsymbol{R} \cdot \boldsymbol{r}$,

which, in one dimension, is equivalent to the last term in Eq. (1). Although we have obtained Eq. (5) classically, the same result follows by transforming the Schrödinger equation to an accelerating reference frame and making a series of gauge transformations.

To study the effect of the last term in Eq. (1) on the electronic energy relaxation rate we calculate the electron self-energy perturbatively. ${ }^{2} \mathrm{We}$

\footnotetext{
${ }^{2} \mathrm{~A}$ rotating-wave analysis shows that perturbation theory is valid here when the energy width $\hbar \gamma$ of the CM translational mode is larger than the interaction energy $g\left|x_{12}\right|$.
} 
assume that $\Delta \varepsilon$ is considerably smaller than $\hbar \Delta \omega$ so that ordinary phonon emission is prohibited (this allows us to set $g_{n \alpha \alpha^{\prime}}=0$ ). However, because the translational motion is that of a macroscopic harmonic oscillator, it is necessary on physical grounds to include damping of that oscillator. We do this by including another term $\Delta H$ in Eq. (1), where $\Delta H$ describes the coupling of the $\mathrm{CM}$ harmonic oscillator to a substrate or to other nanoparticles or both. The leading-order Euclidian self-energy is

$\Sigma(\alpha, i \omega)=-\frac{g^{2}}{\beta} \sum_{\omega_{\mathrm{B}}} \sum_{\alpha^{\prime}}\left|x_{\alpha \alpha^{\prime}}\right|^{2} G_{0}\left(\alpha^{\prime}, \mathrm{i} \omega-\mathrm{i} \omega_{\mathrm{B}}\right) D\left(\mathrm{i} \omega_{\mathrm{B}}\right)$,

where $\beta$ is the inverse temperature, $\omega_{\mathbf{B}}$ is a bosonic Matsubara frequency, $G_{0}(\alpha, i \omega)$ is the noninteracting electron Green's function, and $D\left(\mathrm{i} \omega_{\mathrm{B}}\right)$ is the Fourier transform of a renormalized phonon propagator $\quad D(\tau) \equiv-\langle T[b(\tau)+\bar{b}(\tau)][b(0)+\bar{b}(0)]\rangle$. The precise form of $D\left(\mathrm{i} \omega_{\mathrm{B}}\right)$ depends, of course, on $\Delta H$, and in the absence of a reliable microscopic model for $\Delta H$ we shall use a (retarded) propagator of the form

$D^{\mathrm{R}}(\omega)=\frac{1}{\omega-\Omega+\mathrm{i} \gamma}-\frac{1}{\omega+\Omega+\mathrm{i} \gamma}$,

which has a Lorentzian spectral function of width $\gamma$.

The excited state relaxation rate $\tau^{-1} \equiv$ $-\left.2 \operatorname{Im} \Sigma^{\mathrm{R}}\left(\alpha, \varepsilon_{\alpha}\right)\right|_{\alpha=2}$ at zero temperature is found to be

$\tau^{-1}=\frac{2 \pi g^{2}\left|x_{12}\right|^{2}}{\hbar^{2}} f(\Delta \varepsilon-\hbar \Omega)$,

where $f(\omega) \equiv \gamma / \pi\left(\omega^{2}+\gamma^{2}\right)$ is a Lorentzian function with width $\gamma$. When the energy separation $\Delta \varepsilon$ between two levels is resonant with the frequency $\Omega$ of the translational mode, the relaxation rate becomes

$\tau^{-1}=\frac{4 g^{2}\left|x_{12}\right|^{2}}{\hbar^{2}} \tau_{\mathrm{ph}}$

where $\tau_{\mathrm{ph}} \equiv 1 / 2 \gamma$ is the lifetime of the translational mode. The fact that relaxation occurs even at zero temperature, when the nanoparticle $\mathrm{CM}$ is in its ground state, shows that CM zero-point motion is sufficient to produce a fictitious force.

To establish the experimental observability of this mechanism, we estimate the resonant relaxation rate, given by Eq. (9), for a nanoparticle similar to that studied in Ref. [2]. For a $\mathrm{Y}_{2} \mathrm{O}_{3}$ nanoparticle with diameter $d=15 \mathrm{~nm}$, density $\rho=5.0 \mathrm{~g} / \mathrm{cm}^{-3}$, and mass $M=8.8 \times 10^{-18} \mathrm{~g}$, we find

$\tau^{-1} \approx 1.8 \times 10^{-4}\left(\frac{\left|x_{12}\right|}{a_{\mathrm{B}}}\right)^{2}\left(\frac{\Omega}{1 \mathrm{~cm}^{-1}}\right)^{2} Q \mathrm{~s}^{-1}$,

where $a_{\mathrm{B}}$ is the Bohr radius, $1 \mathrm{~cm}^{-1}$ $=1.8 \times 10^{11} \mathrm{~s}^{-1}$ is one wavenumber in frequency, and $Q \equiv \tau_{\mathrm{ph}} \Omega$ is the quality factor of the CM oscillator. In particular, for $\left|x_{12}\right|=a_{\mathrm{B}}$ and $\Omega=3 \mathrm{~cm}^{-1}$, we predict relaxation rates of

$$
\begin{aligned}
& \tau^{-1} \approx 1.6 \times 10^{-1} \mathrm{~s}^{-1} \quad \text { for } Q=10^{2}, \\
& \tau^{-1} \approx 1.6 \times 10^{3} \mathrm{~s}^{-1} \quad \text { for } Q=10^{6},
\end{aligned}
$$

The second estimate applies to a very weakly damped nanoparticle. It is simple to show that for the $8.8 \times 10^{-18} \mathrm{~g}$ nanoparticle considered here, the maximum $Q$ factor allowed by the perturbative analysis leading to Eq. (8) is about $10^{7}$ (see footnote 2). Although small, these rates are still much larger than the radiative rate between the same levels.

In conclusion, our analysis shows that fictitious forces produce a coupling between the impurity state and the CM motion. If the CM oscillation frequency is near (on the scale $\gamma$ ) to the level separation $\Delta \varepsilon / \hbar$, this effect provides a mechanism for energy relaxation even when conventional phonon emission is prohibited. We find that on resonance the relaxation rate is proportional to the translational mode lifetime $\tau_{\mathrm{ph}}$. This means that relaxation is faster when the translational mode is weakly coupled to its environment, whereas coupling to an appreciably damped CM mode is less effective. Although we do not believe that the mechanism proposed here is responsible for the energy relaxation observed in Ref. [2], these estimates clearly show that this somewhat exotic phenomena is experimentally accessible. 
This work was supported by a Faculty Research Grant awarded by the University of Georgia Research Foundation, and by a Research Innovation Award from the Research Corporation. It is a pleasure to thank William Dennis, Uwe Happek, Coates Johnson, Vadim Markel, and Richard Meltzer for useful discussions.

\section{References}

[1] B. Henderson, G.F. Imbusch, Optical Spectroscopy of Inorganic Solids, Clarendon Press, Oxford, 1989.

[2] H.S. Yang, S.P. Feofilov, D.K. Williams, J.C. Milora, B.M. Tissue, B.S. Meltzer, W.M. Dennis, Physica B 263-264 (1999) 476.

[3] H. Lamb, Proc. Math. Soc. London 13 (1882) 187. 